\title{
Effect of self-stratification on sediment diffusivity in channel flows and boundary layers: a study using direct numerical simulations
}

\author{
S. Dutta ${ }^{1}$, M. I. Cantero ${ }^{3}$, and M. H. Garcia ${ }^{1,2}$ \\ ${ }^{1}$ Dept. of Civil and Environmental Engineering, University of Illinois at Urbana-Champaign, Urbana, USA \\ ${ }^{2}$ Dept. of Geology, University of Illinois at Urbana-Champaign, Urbana, USA \\ ${ }^{3}$ Centro Atómico Bariloche and Instituto Balseiro, Consejo Nacional de Investigaciones Científicas y Técnicas \\ (CONICET) and Comisión Nacional de Energía Atómica (CNEA), San Carlos de Bariloche, Río Negro, \\ Argentina
}

Correspondence to: S. Dutta (dutta5@illinois.edu)

Received: 28 October 2013 - Published in Earth Surf. Dynam. Discuss.: 19 November 2013

Revised: 14 June 2014 - Accepted: 2 July 2014 - Published: 6 August 2014

\begin{abstract}
Sediment transport in nature comprises of bedload and suspended load, and precise modelling of these processes is essential for accurate sediment flux estimation. Traditionally, non-cohesive suspended sediment has been modelled using the advection-diffusion equation (Garcia, 2008), where the success of the model is largely dependent on accurate approximation of the sediment diffusion coefficients. The current study explores the effect of self-stratification on sediment diffusivity using suspended sediment concentration data from direct numerical simulations (DNS) of flows subjected to different levels of stratification, where the level of stratification is dependent on the particle size (parameterized using particle fall velocity $\tilde{V}$ ) and volume-averaged sediment concentration (parameterized using shear Richardson number $R i_{\tau}$ ). Two distinct configurations were explored, first the channel flow configuration (similar to flow in a pipe or a duct) and second, a boundary-layer configuration (similar to open-channel flow). Self-stratification was found to modulate the turbulence intensity (Cantero et al., 2009b), which in turn was found to reduce vertical sediment diffusivity in portions of the domain exposed to turbulence damping. The effect of particle size on vertical sediment diffusivity has been studied in the past by several authors (Rouse, 1937; Coleman, 1970; Nielsen and Teakle, 2004); so in addition to the effect of particle size, the current study also explores the effect of sediment concentration on vertical sediment diffusivity. The results from the DNS simulations were compared with experiments (Ismail, 1952; Coleman, 1986) and field measurements (Coleman, 1970), and were found to agree qualitatively, especially for the case of channel flows. The aim of the study is to understand the effect of stratification due to suspended sediment on vertical sediment diffusivity for different flow configurations, in order to gain insight of the underlying physics, which will eventually help us to improve the existing models for sediment diffusivity.
\end{abstract}

\section{Introduction}

Turbulent mixing and accompanying transport is a prevalent phenomenon in natural and industrial settings. One of the most important transport phenomena in nature is that of sediment, and it can be broadly divided into bedload transport and suspended load transport. In most rivers, the suspended load comprises approximately $80-85 \%$ of the total sediment load, thus playing an important role in morphodynamics of the system. In situ measurement of suspended sediment is still very discontinuous and expensive, so accurate modelling of transport of suspended sediment is essential for correct approximation of the net sediment flux in a river. For the generic case of suspended sediment of constant density and particle size in unsteady turbulent flow, suspended sediment can be modelled using the Reynolds-averaged mass balance 
equation and the appropriate boundary conditions (Garcia, 2008):

$\frac{\partial \bar{c}}{\partial t}+\frac{\partial F_{i}}{\partial x_{i}}=0$, where $F_{i}=\left(u_{i}-V \delta_{i 3}\right) \bar{c}+\overline{u_{i}^{\prime} c^{\prime}}$.

Here $\bar{c}$ is the mean (averaged over turbulence) volumetric concentration of suspended sediment, $c^{\prime}$ is instantaneous fluctuation of sediment concentration, $u_{i}$ is the mean fluid velocity, $u_{i}^{\prime}$ is turbulent fluctuations, $F_{i}$ is the Reynoldsaveraged suspended sediment flux, $V$ is particle settling velocity in quiescent water and $\delta_{i 3}$ is the Kronecker delta. With the assumption of the river/stream flowing at a steady state and being confined in a wide channel, Eq. (1) reduces to (Garcia, 2008)

$\frac{\mathrm{d}}{\mathrm{d} z}\left(\overline{w^{\prime} c^{\prime}}-V \bar{c}\right)=0$

Under typical conditions prevailing in most streams and rivers, the suspended sediment can be safely assumed to be in equilibrium; and combining it with the boundary conditions at free surface, Eq. (2) further reduces to $\overline{w^{\prime} c^{\prime}}-V \bar{c}=$ 0 . The eddy-diffusivity assumption can be used to model $\overline{w^{\prime} c^{\prime}}$; the resulting relationship has been widely used for modelling transport of suspended sediment (Rouse, 1937; Vanoni, 1946):

$K_{z} \frac{\mathrm{d} \bar{c}}{\mathrm{~d} z}+V \bar{c}=0$,

where $K_{z}$ is the vertical sediment diffusivity due to turbulence mixing. Success of the above model depends on the correct estimation of the sediment diffusivity coefficient. Using Prandtl's analogy, and assuming that the logarithmic velocity profile holds for the full depth of the flow, Rouse (1937) derived a formula for $K_{z}$

$\frac{K_{z}}{H u_{*}}=\kappa \frac{z}{H}\left(1-\frac{z}{H}\right)$.

In the above equation, $H$ is depth of the flow, $\kappa$ is the von Karman constant, $u_{*}$ is the bed shear velocity and $z$ is the normal distance from the bed. Even though Prandtl's analogy might not perfectly hold under all circumstances, the above relation (also known as the Rousian formulation for vertical eddy viscosity) has been used extensively in the field of suspended sediment transport. One of the first studies to question the universal applicability of the Rousian formulation was Coleman (1970); he used suspended sediment measurements from lab experiments and field measurements to calculate $K_{z} / H u_{*}$ for sands with different values of $V / u_{*}$. Rearranging Eq. (3) and dividing both sides by $H u_{*}$ gives us the formula used for calculating $K_{z} / H u_{*}$ :

$\frac{K_{z}}{H u_{*}}=-\frac{\overline{\tilde{c}} \tilde{V}}{\mathrm{~d} \overline{\tilde{c}} / \mathrm{d} \tilde{z}}$

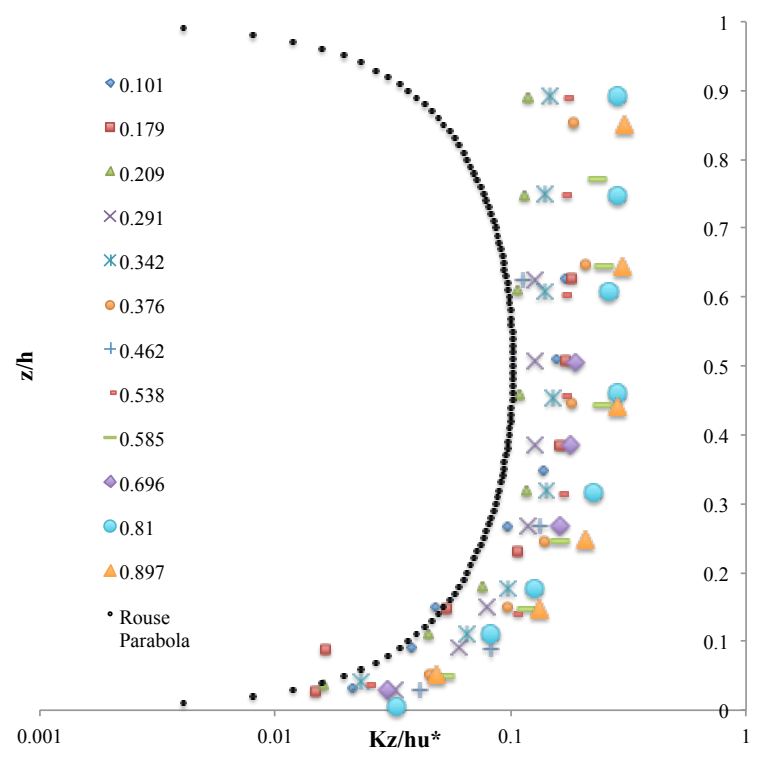

Figure 1. Vertical sediment diffusivity $K_{z} / H u_{*}$ profiles for sediments with different $\tilde{V}=V / u_{*}$. The data has been reproduced from calculations done by Coleman (1970) on field data of Anderson (1942). The generic Rousian profile of kinematic eddy viscosity has also been plotted. For most cases the Rousian profile underestimates sediment diffusivity. There is a trend that vertical sediment diffusivity increases with increase in $\tilde{V}$; but the trend is not obvious for some of the cases plotted above (e.g. between 0.585 and 0.696).

In the above equation $\tilde{V}$ is $V / u_{*}, \tilde{z}$ is $z / H$ and $\overline{\tilde{c}}$ is the mean volumetric suspended sediment concentration normalized by volume-averaged concentration $c^{(v)}$. In his study Coleman (1970) used field data of Anderson (1942) to calculate $K_{z} / H u_{*}$, and the same data has been reproduced here (Fig. 1) along with the Rousian profile calculated using Eq. (4). It can be observed in Fig. 1 that $K_{z} / H u_{*}$ is parabolic only in the lower portion of the domain; also for most cases the Rousian profile underestimates vertical sediment diffusivity. Van Rijn (1984) put forward the idea that the ratio of sediment diffusivity and kinematic eddy diffusivity is always greater than 1 and suggested the use of an empirical coefficient to adjust kinematic eddy diffusivity to match the vertical sediment diffusivity. However, Bennett et al. (1998) attributed the disparity to the use of suspended sediment concentration profiles to calculate sediment diffusivity, and instead recommended the use of direct turbulence measurements. In general, the common consensus has been that the Rousian profile is not an appropriate surrogate for vertical sediment diffusivity.

The Rousian profile, though a very good first approximation, does not capture all the ingrained physics present in the interaction of suspended sediment and the ambient fluid. One of the first one to point out the breakdown of Prandtl's analogy for real sediments was Rouse (1938) through his classic jar experiments. Among the different contributing mechanisms that influence the break down of Prandtl's analogy, one 
of the most prominent one is the inertial effect of relatively large sediment particles (Nielsen, 1992). Further, Nielsen and Teakle (2004) used the finite-mixing-length theory to justify their interpretation of Coleman's (1970) data, in which they point out that vertical sediment diffusivity increases with increase in $V / u_{*}$ (dubbed as the Rouse number, though exact definition of the Rouse number is $\left.V / \kappa u_{*}\right)$. In Fig. 1 data from Coleman (1970) has been reproduced; an obvious trend emerges where $K_{z} / H u_{*}$ for cases with higher $V / u_{*}$ is higher than those with lower $V / u_{*}$. But the aforementioned trend is not universal, and there are cases where the sediment with relatively lower $V / u_{*}$ has higher or almost equal sediment diffusivity when compared with sediment with relatively higher $V / u_{*}$ (e.g. compare the cases with $V / u_{*}$ of 0.585 and 0.696). This may be an artefact of an unrecognized competing mechanism that tends to reduce the vertical sediment diffusivity with increase in $V / u_{*}$.

The hypothesis is that the aforementioned anomaly can be explained if the effect of self-stratification due to suspended sediment on sediment diffusivity is accounted. The settling sediment particles form a continuous concentration profile, with higher concentration near the bottom and lower at the top. This concentration gradient causes stratification in the fluid, and as the suspended sediment particles themselves cause stratification, the phenomenon is also referred to as self-stratification. This concentration gradient is known to modulate turbulence and affect bulk properties of the flow (Cantero et al., 2009b, 2012; Shringarpure et al., 2012). Wright and Parker (2004) showed the importance of sediment-induced stratification in large low-gradient streams/rivers. Smith and McLean (1977) and McLean (1992), among others, proposed the use of simple algebraic closures based on the gradient Richardson number $\left(R i_{\mathrm{g}}\right)$ to take into account the effect of self-stratification on the Rousian profile. Through laboratory experiments, Cellino and Graf (1999) reported the suppression of turbulence due to presence of suspended sediment. In their experiments, they found the estimated momentum and sediment diffusivity to be smaller than the theoretically predicated value (the Rousian profile). Cellino and Graf (2000) also studied the effect of bed-forms on vertical sediment and momentum diffusivity, and found that presence of bed-forms increases the ratio of vertical sediment and momentum diffusivity from less than 1.0 to greater than 1.0 (Graf and Cellino, 2002).

The aim of the present study is to explore the effect of selfstratification on vertical sediment diffusivity under two different configurations: first for channel flows, which is an analogue for flow in a pipe or a duct; and second for a boundarylayer configuration, which is similar to an open-channel flow. For the first portion of the study, we have used steady-state sediment concentration profiles from direct numerical simulations (DNS) of sediment-laden flows. For the DNS, sediment has been modelled using an Eulerian approach with the assumption that the sediment particles do not have any inertia. Though this is not true for larger sediment in na- ture, it is a good assumption for fine sediment. It was also done this way to explore the effect of self-stratification without other mechanisms (like inertial effects; see for example Cantero et al., 2008) coming into play. DNS was done for a constant shear Reynolds number $\left(R e_{\tau}\right)$ but for different levels of self-stratification, which depend on the sediment particle settling velocity (parameterized using $\tilde{V}=V / u_{*}$ ) and volume-averaged suspended sediment concentration (parameterized using shear Richardson number $R i_{\tau}$ ). Traditionally, sediment diffusivity under different circumstances has primarily been studied for the open-channel-like configuration; so the present study also explores it in the channel flow setting. Apart from using data from DNS, data from experiments by Ismail (1952) and Coleman (1986) have been used to study the effect of stratification on sediment diffusivity. The aim of the current study is to extend our understanding of the effects of self-stratification on sediment diffusivity in channel and open-channel-like flows.

\section{Mathematical formulation}

DNS were conducted for a horizontal channel, where the flow is driven by a constant pressure gradient. The constant pressure gradient here is a surrogate for a constant slope in a stream/river that drives the flow, especially for the openchannel-like configuration. Suspended sediment particles are assumed to be of constant size, negligible inertia, and having a constant settling velocity $\tilde{V}$. Eulerian representation has been used to represent the suspended sediment particles, and this has been found to be valid for sediment particles that are small enough (Ferry and Balachandar, 2001). The flow is assumed to be dilute enough that the Boussinesq approximation holds. The set of dimensionless equations used to model the flow is

$$
\begin{aligned}
& \frac{\partial \tilde{u}_{i}}{\partial \tilde{t}}+\tilde{u}_{j} \frac{\partial \tilde{u}_{i}}{\partial \tilde{x}_{j}}=\tilde{G} \delta_{i 1}-\frac{\partial \hat{p}}{\partial \tilde{x}_{i}}+\frac{1}{R e_{\tau}} \frac{\partial^{2} \tilde{u}_{i}}{\partial \tilde{x}_{j} \partial \tilde{x}_{j}}-R i_{\tau} \tilde{c} \delta_{i 3}, \\
& \frac{\partial \tilde{u}_{i}}{\partial \tilde{x}_{i}}=0, \\
& \frac{\partial \tilde{c}}{\partial \tilde{t}}+\left(\tilde{u}_{j}-\tilde{V} \delta_{i 3}\right) \frac{\partial \tilde{c}}{\partial \tilde{x}_{j}}=\frac{1}{S c R e_{\tau}} \frac{\partial^{2} \tilde{c}}{\partial \tilde{x}_{j} \partial \tilde{x}_{j}} .
\end{aligned}
$$

In the above equations, $\tilde{u}_{i}$ is the velocity of the fluid phase, $\tilde{c}$ is the volumetric concentration of the suspended sediment particles. $\tilde{G}$ is the constant streamwise mean pressure gradient driving the flow and has a magnitude equal to 1 and $\hat{p}$ is the pressure field, which is the combination of the dynamic pressure $(\tilde{p})$ and the hydrostatic component due to the suspended sediment. The mathematical formulation used in the present study is exactly the same as the one used by Cantero et al. (2009b) in their study of turbulence modulation due to self-stratification, and additional details about the model can be found in Cantero et al. (2009b). Sediment has been modelled under the Eulerian framework using the advectiondiffusion equation (see Eq. 6c). The diffusion term in Eq. (6c) 
might look out of place, but it serves multiple purposes. Even though the sediment particles are assumed to be big enough that their Brownian motion can be ignored, it is well established that relatively large particles can also diffuse due to long-range hydrodynamic interactions (Mucha and Brenner, 2003) and the diffusive term takes into account the aforementioned mechanism. The diffusion term also provides a way to resuspend sediment from the bed (Garcia and Parker, 1993), while providing numerical stability (Cantero et al., 2009a) to the simulation.

In the above set of equations, all the variables are dimensionless. Velocity has been made dimensionless using average shear velocity $\left(u_{*}\right)$; the parameter used for scaling length is the channel half-height $h$ (where $2 h$ is the height of the channel) and the parameter used for scaling pressure is $\rho_{f} u_{*}^{2}$, where $\rho_{f}$ is ambient fluid density. Equation (6) has four dimensionless numbers, which together define various properties of the flow; shear Reynolds number $\left(R e_{\tau}\right)$, shear Richardson number $\left(R i_{\tau}\right)$, Schmidt number $(S c)$ and the non-dimensional particle fall velocity $(\tilde{V})$. These nondimensional numbers are defined as

$$
\begin{aligned}
& R e_{\tau}=\frac{u_{*} h}{v} \quad R i_{\tau}=\frac{g R c^{(v)} h}{u_{*}^{2}}, \\
& S c=\frac{v}{K_{\mathrm{s}}} \quad \tilde{V}=\frac{V}{u_{*}},
\end{aligned}
$$

where $v$ is the kinematic viscosity, $g$ is acceleration due to gravity, $K_{\mathrm{S}}$ is diffusivity of the sediment particles (this diffusion of sediments arise from their long-range hydrodynamic interaction; see for example Segre et al., 2001), $c^{(v)}$ is the volume-averaged concentration, $R=\rho_{\mathrm{s}} / \rho_{f}-1$ and $\rho_{\mathrm{s}}$ is the density of the sediment particles. In the current study all the DNSs were done for $R e_{\tau}=180$. The shear Reynolds number of the flow was kept constant, as the aim of the study was to understand the effect of self-stratification when the flow remains the same. The shear Richardson number $\left(R i_{\tau}\right)$ is used to parameterize the initial volume-averaged suspended sediment concentration $\left(c^{(v)}\right)$, and $R i_{\tau}$ has been found to play an important role in influencing the final degree of selfstratification (Dutta, 2012). $\tilde{V}$ has also been found to influence the degree of self-stratification by defining the sediment concentration profile among different cases having constant $R i_{\tau}$ (initial sediment concentration) and $R e_{\tau}$ (Cantero et al., 2009b). Dutta (2012) showed that both $\tilde{V}$ and $R i_{\tau}$ have an effect on the final degree of self-stratification, so in the current study both $\tilde{V}$ and $R i_{\tau}$ have been varied to impose different levels of self-stratification. Based on observations made in previous studies (Cantero et al., 2009b), in the present study the Schmidt number $(S c)$ has been kept equal to 1 .

The above-stated governing equations were solved using a dealiased pseudo-spectral code. The setup is exactly the same as the one used by Cantero et al. (2009b), so further details of the exact numerical methods adopted can be found there. Dimensions of the rectangular domain used for the numerical simulations were $\tilde{L}_{x}=4 \pi, \tilde{L}_{y}=4 \pi / 3$ and $\tilde{L}_{z}=2$, and the domain was discretized using a computational grid having $N_{x}=96, N_{y}=96$ and $N_{z}=97$ nodes in the $x, y$ and $z$ (wall-normal) directions respectively. The grid size is uniform in the longitudinal and transverse directions, and in terms of wall units $\left(\tilde{z}^{+}\right)$they are 23.562 and 7.854 respectively. For the wall-normal direction a Chebyshev expansion with Gauss-Lobatto quadrature points has been used. This allows for a very high resolution near the boundaries and relatively lower resolution at the centre of the domain. In terms of wall units, the distance between two nodes is 0.0964 for the nodes near the wall and 5.889 at the centre of the domain. Cantero et al. (2009b) had found the aforementioned computation-grid resolution sufficient for capturing all the relevant flow statistics. Periodic boundary conditions were used in the longitudinal and transverse directions. The top and bottom walls of the domain were assumed to be smooth; and depending on the configuration simulated, a no-slip or slip boundary condition was imposed on the fluid phase at the top wall. At the bottom wall a no-slip condition was employed for all simulations. Sediment particles were assumed to be fine enough to have zero net deposition; thus a boundary condition was imposed, which instantly re-entrains all settled sediment particles. For the channel flow configuration, the imposed boundary conditions are mathematically represented as

$\tilde{u}_{i}=0$ at $\tilde{z}=-1$ and $\tilde{z}=1$,

$\tilde{c} \tilde{V}+\frac{1}{\operatorname{Re}_{\tau} S c} \frac{\partial \tilde{c}}{\partial \tilde{z}}=0$ at $\tilde{z}=-1$ and $\tilde{z}=1$.

For the open-channel-like configuration (boundary-layer configuration), the imposed boundary conditions are

$$
\begin{aligned}
& \tilde{u}_{i}=0 \text { at } \tilde{z}=-1 \text { and } \frac{\partial \tilde{u}}{\partial \tilde{z}}=\frac{\partial \tilde{v}}{\partial \tilde{z}}=\tilde{w}=0 \text { at } \tilde{z}=1, \\
& \tilde{c} \tilde{V}+\frac{1}{\operatorname{Re}_{\tau} S c} \frac{\partial \tilde{c}}{\partial \tilde{z}}=0 \text { at } \tilde{z}=-1 \text { and } \tilde{z}=1 .
\end{aligned}
$$

The boundary condition imposed for suspended sediment allows the net amount of sediment in suspension to remain constant throughout the simulation. When integrated over time, the aforementioned condition allows the flow to reach a statistically steady state (Cantero et al., 2009b).

\section{Results}

Sixteen DNS simulations were run for the present study. They were all run for the same shear Reynolds number of 180 , but different particle fall velocities $(\tilde{V})$ and shear Richardson number $\left(R i_{\tau}\right)$. All the simulated cases have been listed in Table 1 . The simulations can be broadly divided into two parts: twelve that were done with the channel flow configurations and four done with the boundary-layer configuration. The sediment concentration profiles obtained from the simulations were used in conjunction with Eq. (5) to obtain sediment diffusivity profiles $\left(K_{z}\right)$. In order to quantify the 
Table 1. The table lists all the cases of direct numerical simulations used in the current study. All the simulations have the same $R e_{\tau}$. Cases 1-12 correspond to the simulations for channel configuration and 13-16 correspond to the simulations for open-channel-like configuration. Case 1 corresponds to the case with no sediment in suspension, and was simulated to compare with the self-stratified cases.

\begin{tabular}{crrrl}
\hline Case & $R e_{\tau}$ & $\tilde{V}=V / u_{*}$ & $R i_{\tau}$ & Configuration \\
\hline 1 & 180 & 0 & 0 & channel \\
2 & 180 & 0.005 & 18 & channel \\
3 & 180 & 0.01 & 18 & channel \\
4 & 180 & 0.015 & 18 & channel \\
5 & 180 & 0.02 & 18 & channel \\
6 & 180 & 0.025 & 18 & channel \\
7 & 180 & 0.03 & 18 & channel \\
8 & 180 & 0.025 & 1 & channel \\
9 & 180 & 0.025 & 10 & channel \\
10 & 180 & 0.025 & 15 & channel \\
11 & 180 & 0.025 & 20 & channel \\
12 & 180 & 0.025 & 22 & channel \\
13 & 180 & 0.025 & 1 & B. layer \\
14 & 180 & 0.025 & 10 & B. layer \\
15 & 180 & 0.025 & 15 & B. layer \\
16 & 180 & 0.025 & 18 & B. layer \\
\hline
\end{tabular}

sediment diffusivity profiles, three different parameters were defined and calculated for each of the sediment diffusivity profiles. The parameters (also referred to as shape factors) are mean sediment diffusivity $K_{z \mu}$, variance within the sediment diffusivity profile $K_{z \sigma}$ and skewness of the sediment diffusivity profile $K_{z \gamma}$. Three parameters were used in order to take care of ambiguity that may arise due to two different profiles having almost the same mean or/and variance. The three parameters have been defined below.

$$
\begin{aligned}
K_{z \mu} & =\int_{0}^{H} \frac{K_{z}(z)}{H} \mathrm{~d} z \\
K_{z \sigma} & =\left(\int_{0}^{H} \frac{\left(K_{z}-K_{z \mu}\right)^{2}}{H} \mathrm{~d} z\right)^{1 / 2} \\
K_{z \gamma} & =\frac{\int_{0}^{H} \frac{\left(K_{z}-K_{z \mu}\right)^{3}}{H} \mathrm{~d} z}{\left(\int_{0}^{H} \frac{\left(K_{z}-K_{z \mu}\right)^{2}}{H} \mathrm{~d} z\right)^{3 / 2}}
\end{aligned}
$$

In the equations defined above, $H$ corresponds to the total depth of the flow.

\subsection{Channel flow configuration}

Among the twelve simulations done for the channel flow configuration, the first one was done without any suspended sed-
Table 2. The table lists all the cases of direct numerical simulations used in the current study along with $\tilde{V} R i_{\tau}$ for each of the cases. $\tilde{V} R i_{\tau}$ is a parameter which represents the level of self-stratification; and a higher value of $\tilde{V} R i_{\tau}$ corresponds to a higher level selfstratification if $R e_{\tau}$ remains constant. Cases 1-12 correspond to the simulations for channel configuration and 13-16 correspond to the simulations for open-channel-like configuration. The corresponding sediment diffusivity profiles were quantified using Eq. (10), and the calculated parameters $K_{z \mu}, K_{z \sigma}, K_{z \gamma}$ have also been listed in the table. $K_{z \mu}$ parameterizes the mean sediment diffusivity, $K_{z \sigma}$ parameterizes the variance in the sediment diffusivity profile and $K_{z \gamma}$ parameterizes the skewness of the sediment diffusivity profile.

\begin{tabular}{ccccr}
\hline Case & $\tilde{V} R i_{\tau}$ & $K_{z \mu}$ & $K_{z \sigma}$ & $K_{z \gamma}$ \\
\hline 2 & 0.090 & 0.0607 & 0.0231 & -0.9897 \\
3 & 0.180 & 0.0485 & 0.0212 & -0.3191 \\
4 & 0.270 & 0.0412 & 0.0231 & 0.3853 \\
5 & 0.360 & 0.0426 & 0.0310 & 0.6639 \\
6 & 0.450 & 0.0602 & 0.0516 & 0.3016 \\
7 & 0.540 & 0.0610 & 0.0523 & 0.2976 \\
8 & 0.025 & 0.0724 & 0.0272 & -1.3216 \\
9 & 0.250 & 0.0452 & 0.0272 & 0.5594 \\
10 & 0.375 & 0.0618 & 0.0525 & 0.2931 \\
11 & 0.500 & 0.0592 & 0.0511 & 0.31162 \\
12 & 0.550 & 0.0582 & 0.0507 & 0.3255 \\
13 & 0.025 & 0.1927 & 0.0882 & -0.7991 \\
14 & 0.25 & 0.1430 & 0.0716 & -0.5649 \\
15 & 0.375 & 0.1278 & 0.0676 & -0.4417 \\
16 & 0.450 & 0.1204 & 0.0653 & -0.3882 \\
\hline
\end{tabular}

iment. A set of simulations (set1) was done for constant shear Richardson number but increasing $\tilde{V}$. This set is equivalent to the situation where the initial volume-averaged suspended sediment concentration is constant but the sediment particle size increases. The other set of simulations (set2) done for the same configuration is with a constant $\tilde{V}$ equal to 0.025 but increasing shear Richardson number. This set is equivalent to the situation where the particle size of sediment is constant but the initial volume-averaged suspended sediment concentration increases. For both set 1 and set 2 increase in $\tilde{V}$ or $R i_{\tau}$ while keeping the other parameters constant results in an increase in the degree of stratification. Dutta (2012) showed that increase in either $\tilde{V}$ or $R i_{\tau}$ increases the degree of self-stratification caused by suspended sediment and the extent to which a flow will stratify depends on the parameter $\tilde{V} R i_{\tau}$. Cantero et al. (2012) made similar observations for turbidity currents.

In Figs. 2 and 3 the mean streamwise velocity, steady-state sediment concentration profile and normalized wall-normal turbulence intensity for set 1 and set 2 have been plotted. For both sets, the increase in degree of self-stratification leads to increase in bulk streamwise velocity of the flow. The flow also becomes asymmetric, with the velocity maximum getting skewed towards the channel bottom. One of the effects of the self-stratification is reduction of the bottom drag. The 

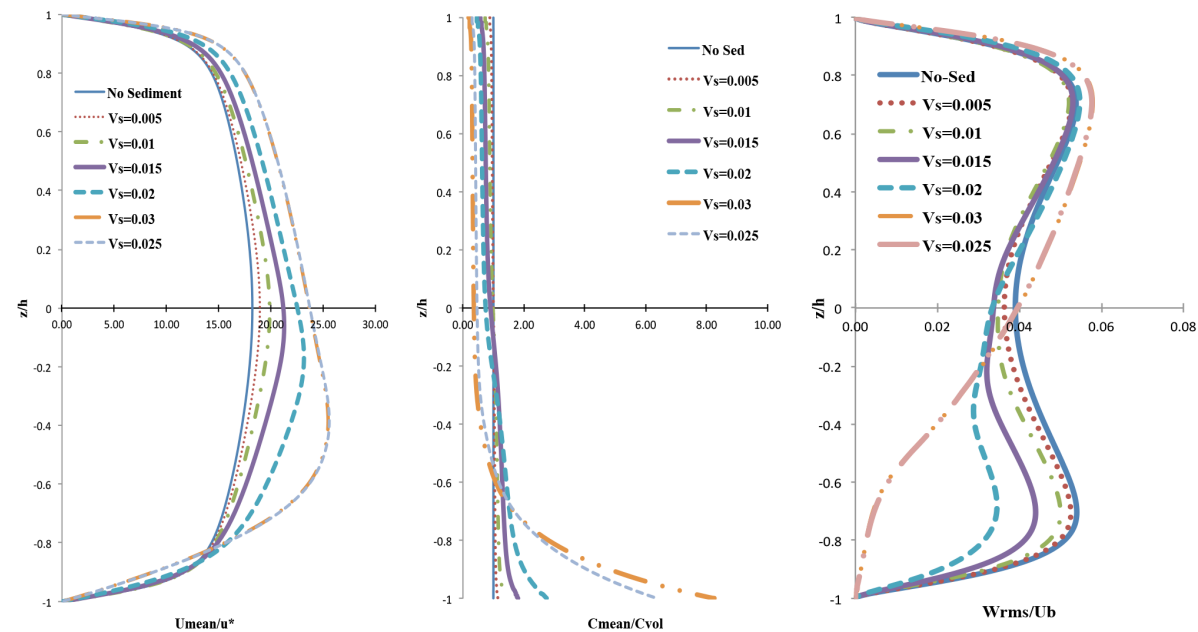

Figure 2. Results from the DNSs in a channel flow setting, for increasing $\tilde{V}$ and $R i_{\tau}=18$. The mean streamwise velocity and asymmetry of the flow increase with increase in $\tilde{V}$. Increase in $\tilde{V}$ increases the degree of self-stratification of the flow; this leads to increase in the sediment concentration gradient and higher amount of turbulence damping near the channel bottom. In the channel, normalized turbulence intensity $\left(W_{\mathrm{rms}} / U_{b}\right)$ is modulated, with a decrease in the lower half of the channel and slight increase in the upper half of the channel.
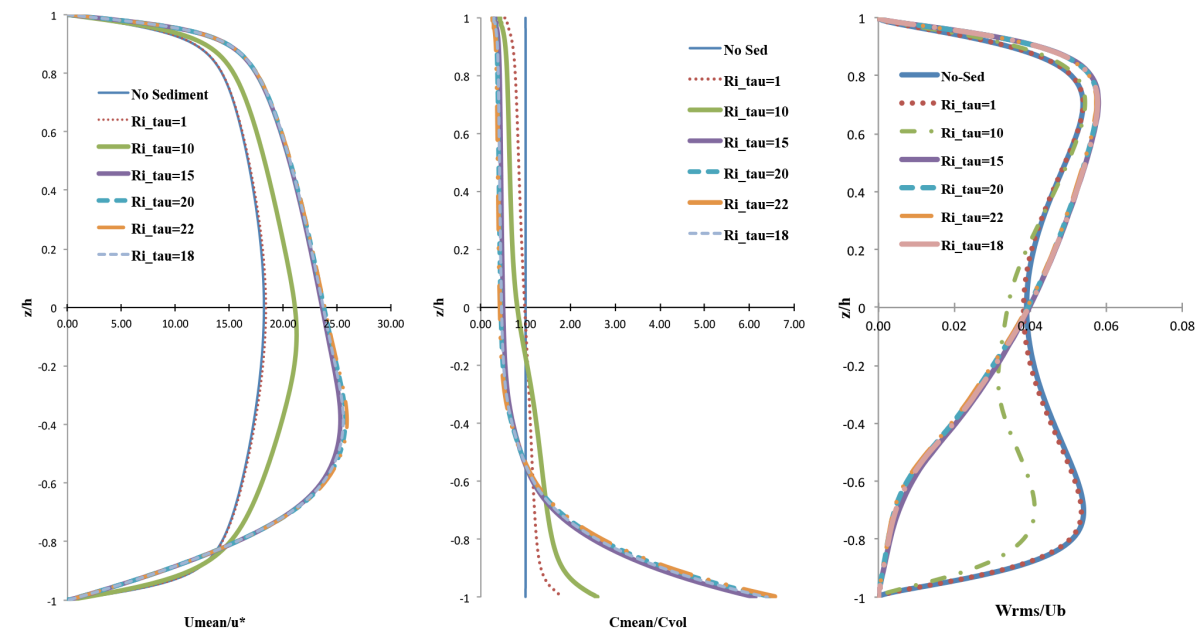

Figure 3. Results from the DNSs in a channel flow setting, for increasing $R i_{\tau}$ and $\tilde{V}=0.025$. The mean streamwise velocity and asymmetry of the flow increase with increase in $R i_{\tau}$. Increase in $R i_{\tau}$ increases the degree of self-stratification of the flow; this leads to increase in the sediment concentration gradient and higher amount of turbulence damping near the channel bottom. In the channel, normalized turbulence intensity $\left(W_{\mathrm{rms}} / U_{b}\right)$ is modulated, with a decrease in the lower half of the channel and slight increase in the upper half of the channel.

decrease in drag results in an increase in the flow discharge in the channel, as the force driving the flow remains the same (the constant unit pressure gradient $\tilde{G}$ ). Even though the bulk streamwise velocity increases, turbulence intensity $\left(W_{\mathrm{rms}}\right)$ in the channel decreases, especially near the channel bottom. The reduction in bottom drag is connected to the reduction of turbulence activity near the bottom of the channel. Reduced turbulence activity in turn reduces the Reynolds stress, thus eventually reducing the total drag at the bottom. Flow in the channel at different levels of self-stratification can be clearly divided into two regimes: first regime (for $\tilde{V} R i_{\tau} \leq 0.36$ ) in which the turbulence near the bottom of the channel is damped but the flow in general is still turbulent. Second regime (for $\tilde{V} R i_{\tau} \geq 0.45$ ) in which turbulence near the bottom of the channel is almost completely suppressed, but turbulence intensity in the upper half of the channel is slightly more than the case with no suspended sediment. Turbulent activity near the top wall is maintained due to lack of stratification in that region. The small increase in turbulent activity is due to the aforementioned increase in flow discharge. The steady-state sediment concentration profiles in Figs. 2 and 3 were used with Eq. (5) to calculate vertical sediment diffusivity profiles (Fig. 4) for all the cases in set 1 and set2. Sediment diffusivity profiles reflect the observable trends of the 
turbulence intensity profiles in Figs. 2 and 3. The aforementioned similarity is along the expected lines because the exact definition of vertical sediment diffusivity is nothing but a surrogate used to model the vertical sediment flux due to turbulence $\left(\overline{w^{\prime} c^{\prime}}\right)$. So wherever turbulence is damped, $K_{z} / H u_{*}$ decreases and wherever turbulence increases $K_{z} / H u_{*}$ also increases. The effect of self-stratification on sediment diffusivity is similar to the effect it has on turbulent intensity. The calculated sediment diffusivity profiles (Fig. 4) were quantified using Eq. (10a-c), and the parameters $K_{z \mu}, K_{z \sigma}$ and $K_{z \gamma}$ have been listed in Table 2. The mean sediment diffusivity shows a counterintuitive trend, initially mean diffusivity $\left(K_{z \mu}\right)$ decreases with increase in stratification $\left(\tilde{V} R i_{\tau}\right)$ but then it suddenly increases and then starts to decrease again (see Fig. 5). The sudden increase is associated with increase of sediment diffusivity in the upper part of the domain. The presence of a top wall which continues to pump turbulent energy into the system, even after the flow at the bottom has relaminarized, is responsible for the variation in the expected trend of decrease of mean sediment diffusivity with increase in stratification, which can be observed in Fig. 5 for the case of open-channel flow. $K_{z \mu}$ for the open-channel flow cases was obtained from DNS simulations that are discussed in the next section. The above-mentioned trend is also reflected in the parameter describing the variation of sediment diffusivity within each profile $\left(K_{z \sigma}\right)$. The trends reflected by all the parameters clearly show the presence of two distinct regimes in the flow. Additionally, the normalized sediment concentration profiles of the simulated cases were plotted with the corresponding analytically obtained suspended sediment concentration profiles. The sediment concentration profiles were normalized using a reference concentration at a height of $b=0.05 H$, where $H$ is the total depth of the channel. For the boundary-layer (open-channel-like) configuration, which will be discussed in the next section, the analytical sediment concentration is given by the well-known Rouse-Vanoni-Ippen suspended sediment distribution (Garcia, 2008):

$$
\frac{\bar{c}}{\bar{c}_{b}}=\left[\frac{(H-z) / z}{(H-b) / b}\right]^{V / u^{*} \kappa},
$$

where $\bar{c}_{b}$ is the reference sediment concentration that is the sediment concentration at a height $b$, and $b$ as defined above. $H$ is the total depth of the flow and rest of the parameters are as previously defined. For the channel flow configuration, a similar relationship was not available. Thus a relationship for suspended sediment concentration in a channel was derived using Eq. (5), an approximate relationship for eddy viscosity in a pressure-driven-channel flow, and the appropriate boundary conditions (Schlichting and Gersten, 2000). The derived relationship for sediment concentration is

$$
\begin{aligned}
& \frac{\bar{c}}{\bar{c}_{b}}=\exp \left[-\frac{V}{u^{*} \kappa}\left\{\ln \left(\frac{z /(z-2 H)}{b /(b-2 H)}\right)+\sqrt{8}\right.\right. \\
& \left.\left.\left(\tan ^{-1}\left(\frac{\sqrt{2}(z-H)}{H}\right)-\tan ^{-1}\left(\frac{\sqrt{2}(b-H)}{H}\right)\right)\right\}\right] .
\end{aligned}
$$

In the above relationship $H$ is the channel half depth and the rest of the parameters are as earlier. Equation (12) was used along with the sediment concentration profiles from the DNS simulations to plot Fig. 6. The normalized sediment concentration profile predicated by Eq. (12) was found to match very well with the DNS-predicted normalized sediment concentration profiles for the cases having relatively small stratification $\left(\tilde{V} R i_{\tau}<0.1\right)$. The performance of Eq. (12) deteriorated with increase in $\tilde{V} R i_{\tau}$, so it clearly shows that the relationship is not able to accommodate the effect self-stratification might have on the suspended sediment concentration profiles, especially the one induced by increase in the concentration of suspended sediment.

\subsection{Boundary-layer configuration}

Four numerical simulations were done for the boundarylayer configuration. The boundary-layer configuration is similar to the open-channel configuration but not exactly the same. Like the open-channel configuration, a slip boundary condition is imposed at the top wall for the fluid phase. In Fig. 7, bulk streamwise velocity, steady-state sediment concentration profile and normalized turbulence intensity have been plotted. The four simulations for the open-channel-like configuration have the same particle fall velocity $(\tilde{V})$ but increasing shear Richardson number. Similar to the channel flow configuration, bulk streamwise velocity was found to increase with increase in shear Richardson number. Turbulence intensity was found to decrease with increase in shear Richardson number, and unlike the channel flow configuration where turbulence intensity decreases in the lower half of the channel and increases in the upper half; turbulence intensity was found to decrease throughout the channel. Though, the extent of damping in the upper half was found to be slightly more than the extent of damping in the lower half of the boundary layer. The steady-state sediment concentration profiles in Fig. 7 were used to calculate vertical sediment diffusivity profiles (Fig. 8) for the four cases simulated. Reflecting the trend shown by turbulence intensity, vertical sediment diffusivity was found to decrease with increase in level of self-stratification. The sediment diffusivity profiles were quantified using Eq. (10) and the parameters $K_{z \mu}, K_{z \sigma}$ and $K_{z \gamma}$ have been listed in Table 2. Unlike the channel flow cases where the trend was counterintuitive, the mean sediment diffusivity $\left(K_{z \mu}\right)$ and variation $\left(K_{z \sigma}\right)$ decreases with increase in stratification (see Fig. 5). Additionally, the normalized sediment concentration profiles of the simulated cases were plotted (see Fig. 8) 

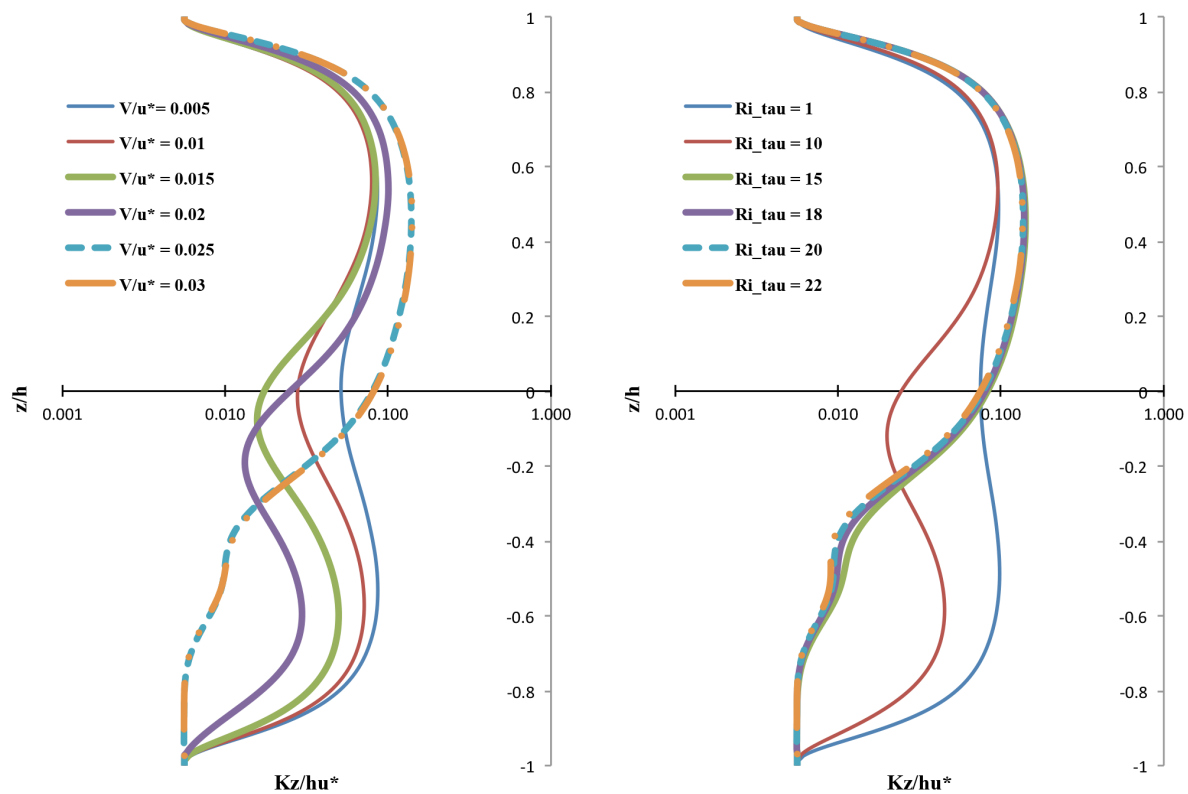

Figure 4. The trend for vertical sediment diffusivity mirrors the trend found for turbulence intensity. An increase in degree of selfstratification is found to decrease sediment diffusivity in the lower half of the channel and increase sediment diffusivity in the upper half of the channel. This is not completely unexpected because mixing of suspended sediment is primarily dependent on turbulence in the flow.

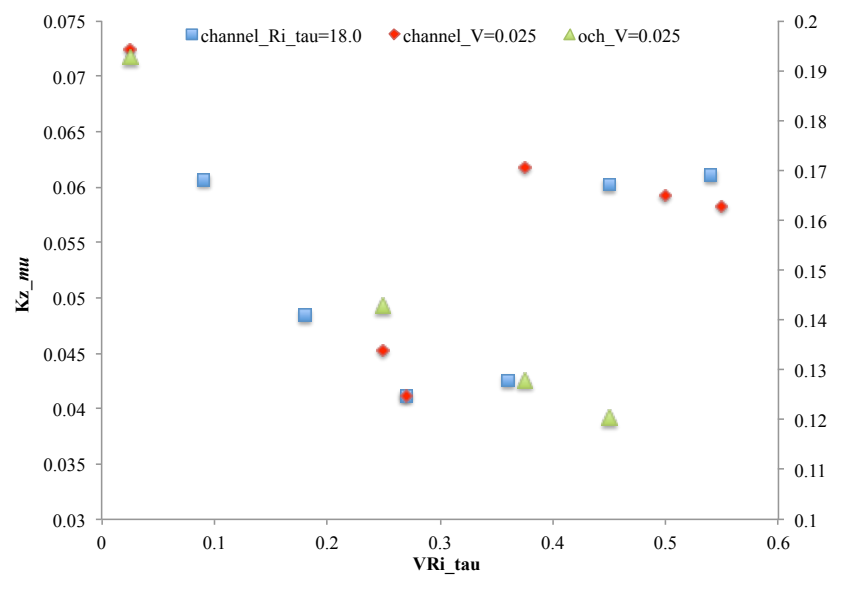

Figure 5. Mean sediment diffusivity $\left(K_{z \mu}\right)$ calculated from all the DNS simulations has been plotted against the corresponding level of stratification $\left(\tilde{V} R i_{\tau}\right) . K_{z \mu}$ for the boundary-layer cases (openchannel-like configuration) has been plotted on the secondary $y$ axis. $K_{z \mu}$ for the open-channel cases decrease monotonically with increase in stratification $\left(\tilde{V} R i_{\tau}\right)$. The aforementioned trend is not reflected in the channel flow configuration. $K_{z \mu}$ for the channel flow configuration has been plotted on the primary $y$ axis, and show a counterintuitive trend of first decreasing, then increasing and then again decreasing with increase in $\tilde{V} R i_{\tau}$.

with the corresponding analytically obtained suspended sediment concentration profile using the Rouse-Vanoni-Ippen relationship (Eq. 11). The normalized sediment concentration profile predicted by the Rouse-Vanoni-Ippen relation- ship was found to match well with the DNS simulations for relatively lower stratification, and the difference increased appreciably with increase in stratification. In the next section the larger implication of the results presented in the previous sections will be discussed. Additionally, the trends observed in the DNS results will be compared with data from lab experiments and field measurements.

\section{Discussion}

In the preceding sections we saw how self-stratification due to suspended sediments can reduce sediment diffusivity in a flow. All the discussed results were on the basis of highresolution numerical simulations, which were set up to exclusively capture the effect of self-stratification on sediment diffusivity. In order to vet our hypothesis further, we have compared our results with experimental observations of Ismail (1952) and Coleman (1986).

Ismail (1952) conducted a series of experiments in a closed rectangular channel. The aim of the experiments was to understand the transfer mechanism of turbulence and its interaction with suspended sediment. The rectangular closedchannel setup is similar to the DNS simulations for the channel flow configuration. Cases 74, 75, 76 and 78 from Ismail (1952) have been used in the current study. All the cases for which suspended sediment concentration profiles were available in Ismail (1952) had dunes at the bottom of the channel, whereas in the DNS simulations the walls were smooth. The presence of the dunes plays an important role, as they can enhance turbulence near the bottom of the channel 

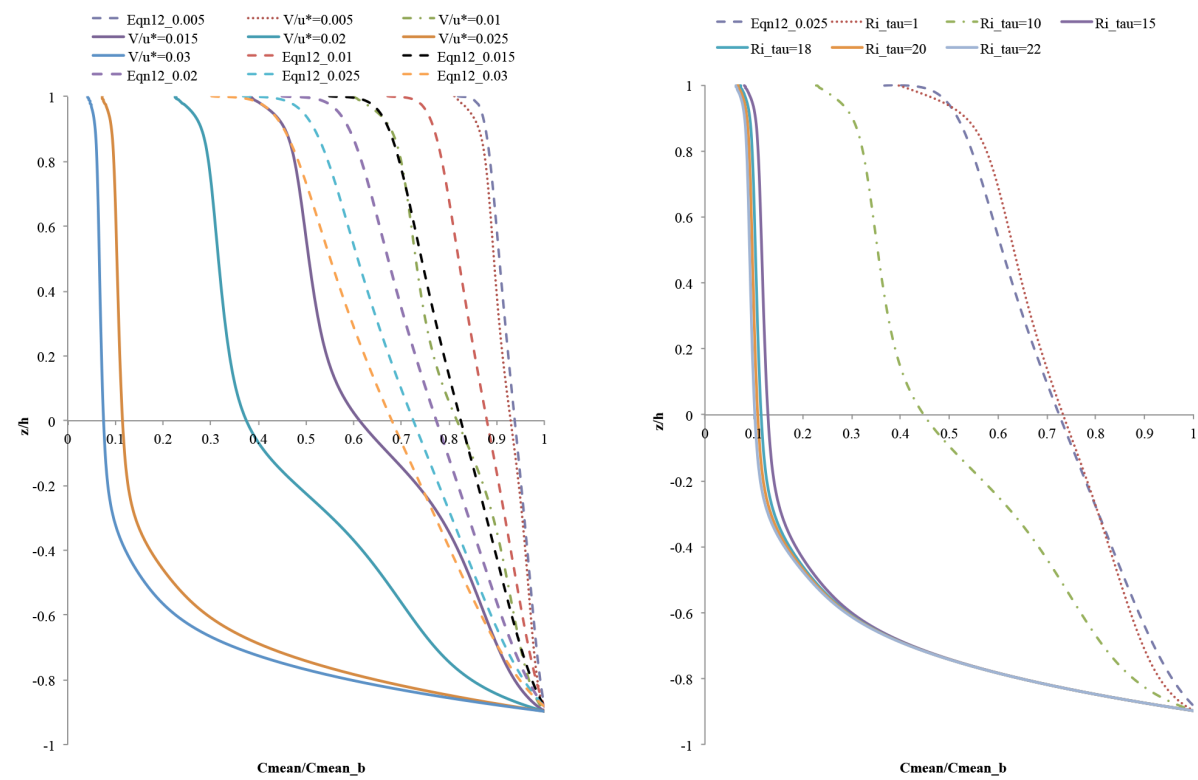

Figure 6. Normalized sediment concentration profiles from DNS simulations of the channel flow have been plotted with the sediment profile obtained using Eq. (12). The results from the DNS simulations matched very well with the derived relationship (Eq. 12) for relatively lower stratification $\left(\tilde{V} R i_{\tau}<0.1\right)$.
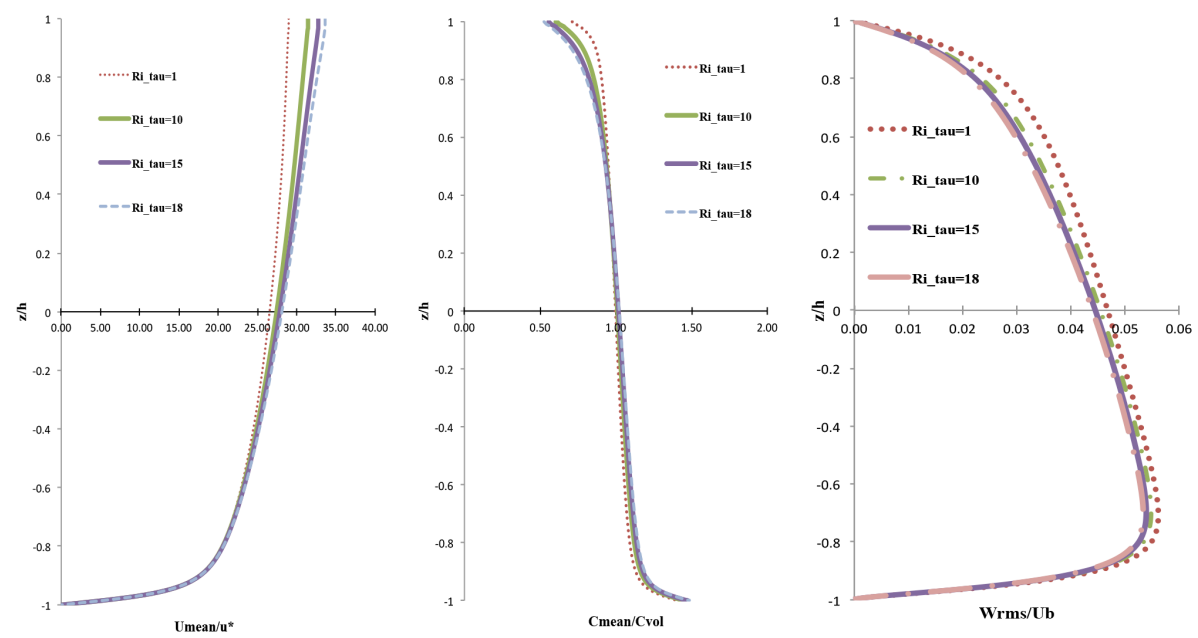

Figure 7. Results from the DNS simulations in a boundary-layer (open-channel-like) setting, for increasing $R i_{\tau}$ and $\tilde{V}=0.025$. Mean streamwise velocity of the flow increases with increase in $R i_{\tau}$. An increase in $R i_{\tau}$ increases the degree of self-stratification of the flow; this leads to an increase in the sediment concentration gradient and higher amount of turbulence damping through out the domain. Normalized turbulence intensity $\left(W_{\mathrm{rms}} / U_{b}\right)$ is damped throughout the boundary layer but the level of suppression is slightly higher in the upper half.

(Ismail, 1952; Cellino and Graf, 2000). But as the comparison between the numerical and the experimental results is strictly qualitative, the effect of the dunes has been neglected. Mean streamwise velocity profiles for cases with (case 117) and without sediment (case 5) have also been reproduced from Ismail (1952), in order to compare the effect suspended sediment has on mean streamwise velocity. Figure 9a reflects the trend shown for streamwise velocity in the DNS results. Compared with the case without sediment, we clearly see in the case with sediment that streamwise velocity in the upper half of the channel increases whereas in the lower half decreases. Suspended sediment concentration profiles for cases 74-76 and 78 were used to calculate the sediment diffusivity profiles (Fig. 9b). All the cases used in the present study have been listed in Table 3; along with their corresponding shear Reynolds number, $R i_{\tau}, \tilde{V}$ and $\tilde{V} R i_{\tau}$. Amongst the cases plotted in Fig. 9, case 74 has the highest level of self-stratification $\left(\tilde{V} R i_{\tau}\right)$ and case 78 the lowest. And moving from case 78 to 74 , vertical sediment diffusivity decreases in the lower half of the channel and increases in upper half of the channel. 

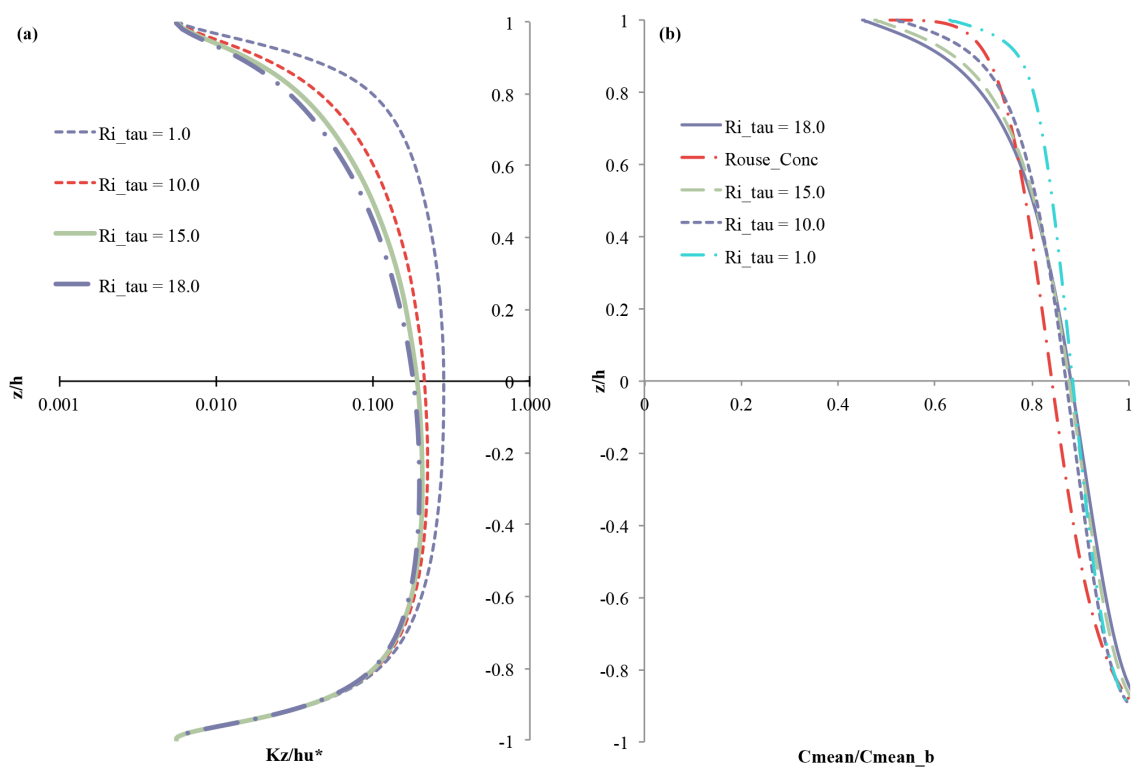

Figure 8. In (a), the trend for vertical sediment diffusivity mirrors the trend found for turbulence intensity. An increase in the degree of self-stratification is found to decrease sediment diffusivity in the boundary layer. The extent to which sediment diffusivity decreases in the upper half of the boundary layer is slightly higher than the lower half of the boundary layer. This is not completely unexpected because mixing of suspended sediment is primarily dependent on turbulence in the flow. In (b), normalized sediment concentration profiles from DNS simulations have been plotted along with the sediment profile obtained using the Rouse-Vanoni-Ippen relationship.
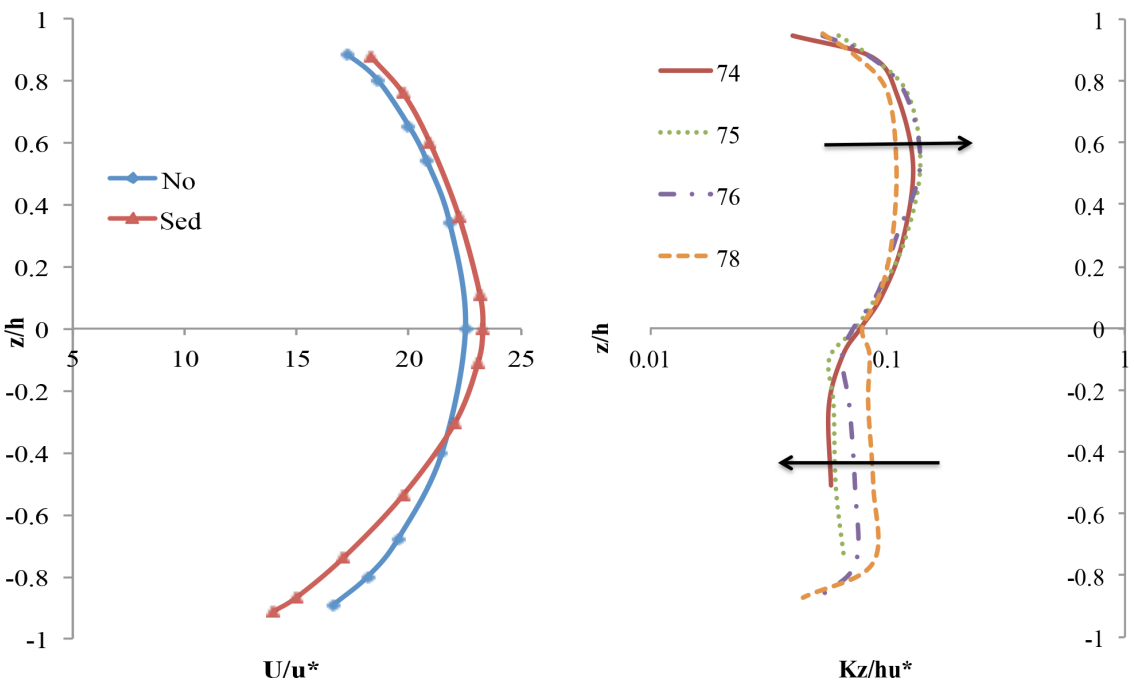

Figure 9. Streamwise velocity for cases 5 and 117 have been reproduced from Ismail (1952). Case 5 has no sediment in suspension and case 117 has suspended sediment. Vertical sediment diffusivity for cases 74-76, and 78 was calculated from sediment concentration profiles from experiments performed by Ismail (1952). With an increase in $\tilde{V} R i_{\tau}$, vertical sediment diffusivity in the channel slightly increases in the top half of the channel and decreases in the lower half of the channel; this completely agrees with the DNS results.

The trend shown by sediment diffusivity calculated from the experimental data of Ismail (1952) concur with the trend observed in the DNS results (Fig. 4). The parameters $K_{z \mu}, K_{z \sigma}$ and $K_{z \gamma}$ were calculated using the sediment diffusivity profiles in Fig. 9 and Eq. (10) and have been listed in Table 4. The mean sediment diffusivity $\left(K_{z \mu}\right)$ showed a trend similar to the one observed for the DNS results.
For comparing the boundary-layer (open-channel-like) case, suspended sediment concentration profiles published by Coleman (1986) were used. Coleman studied the effect of suspended sediment on the velocity distribution of an open-channel flow. The effect of suspended sediment on the streamwise velocity is similar to the effect observed in our DNS results (refer to Fig. 1 in Coleman, 1986). Coleman 
Table 3. List of all the experiments of Ismail (1952) used in the present study.

\begin{tabular}{rcccc}
\hline Case & $R e_{\tau}$ & $\tilde{V}=V / u_{*}$ & $R i_{\tau}$ & $\tilde{V} R i_{\tau}$ \\
\hline 74 & 1409 & 0.25 & 3.629 & 0.90723 \\
75 & 1546 & 0.197 & 4.426 & 0.87192 \\
76 & 1978 & 0.176 & 3.192 & 0.56179 \\
78 & 2698 & 0.133 & 1.729 & 0.22996 \\
5 & 1768 & 0 & 0 & 0 \\
117 & 2188 & 0.359 & 2.926 & 1.05043 \\
\hline
\end{tabular}

Table 4. List of the parameters $K_{z \mu}, K_{z \sigma}$, and $K_{z \gamma}$, calculated from the sediment diffusivity profiles of the cases listed in Table 3.

\begin{tabular}{ccccr}
\hline Case & $\tilde{V} R i_{\tau}$ & $K_{z \mu}$ & $K_{z \sigma}$ & $K_{z \gamma}$ \\
\hline 74 & 0.90723 & 0.0907 & 0.0276 & -0.0819 \\
75 & 0.87192 & 0.0893 & 0.0302 & 0.4050 \\
76 & 0.56179 & 0.0896 & 0.0257 & 0.6515 \\
78 & 0.22996 & 0.0898 & 0.0142 & -0.9668 \\
\hline
\end{tabular}

found the presence of suspended sediment slightly decreased the streamwise velocity near the bottom of the channel and slightly increased it in the upper half of the channel. An increase in streamwise velocity in the upper portion of the boundary layer is also consistent with observations by Barenblatt and Golitsyn (1974) for "mature dust storms". For the present study, we only used the suspended sediment concentration profiles for sediment with a diameter $(D)$ of $0.210 \mathrm{~mm}$. Coleman, in his experiments, used sediment of three different mean diameters, but the hydraulic condition (constant slope and discharge) was kept constant. For each sediment size, sediment was added to the flow till the amount of sediment suspended in the flow reached its maximum (also called the capacity condition). So, for a particular sediment size and flow condition, there are suspended sediment profiles for different net sediment concentrations. The sediment concentration profiles were used to calculate vertical sediment diffusivity profiles (Fig. 10). The cases used for the present study have been listed in Table 5, along with the corresponding $\tilde{V}, R i_{\tau}$ and $\tilde{V} R i_{\tau} . \tilde{V} R i_{\tau}$ increases from case 1 to case 5. In Fig. 10 vertical sediment diffusivity decreases with increase in $\tilde{V} R i_{\tau}$, especially if the difference between case 1 and case 5 is observed; though it is noticeable that the trend of decreasing sediment diffusivity with increase in $\tilde{V} R i_{\tau}$ is not monotonic. This may be attributed to two or more competing mechanisms trying to influence the sediment diffusivity in opposite directions. For example, it is known that depending on the size of the sediment particles in suspension, suspended sediment can increase turbulent kinetic energy (Niño and Garcia, 1998) of the flow, which can then lead to higher sediment diffusivity; whereas increase in self-stratification tends to lower sediment diffusivity. Another factor that might be contributing to this slight incon-

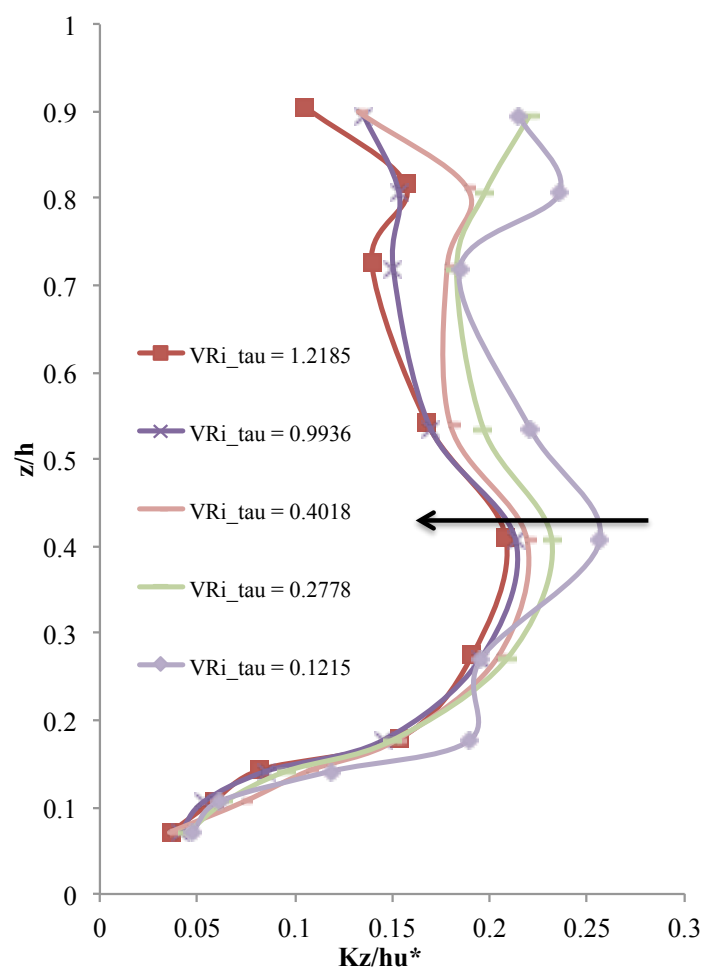

Figure 10. Vertical sediment diffusivity was calculated using sediment concentration profiles from experiments performed by Coleman (1986). With an increase in $\tilde{V} R i_{\tau}$ (cases 1-5), the vertical sediment diffusivity in the open-channel flow decreases.

sistency of the trend in the present case is irregularity of the $\tilde{V}$ values for different cases (refer to Table 5). Even though theoretically all the cases should have the same $\tilde{V}$, the experimental observations actually have some inconsistencies (we checked a copy of the actual data set of N. L. Coleman). As change in $\tilde{V}$ has an influence on the mixing length of the fluid (Nielsen and Teakle, 2004), these small inconsistencies in $\tilde{V}$ might be obfuscating the expected trend of decrease of sediment diffusivity with an increase in $\tilde{V} R i_{\tau}$. Apart from the above-mentioned issue with the size of sediment in suspension, there are inherent uncertainties in estimation of sediment diffusivity, which stem from calculation of shear velocity $u^{*}$ and correct measurement of the sediment concentration. The calculated values of $u^{*}$ in each of the experiments fall within $2.5 \%$ of each other, but this variation was not found to cause any substantial change to the calculated values of sediment diffusivity. The parameters $K_{z \mu}, K_{z \sigma}$ and $K_{z \gamma}$ were also calculated (Table 6); as expected, $K_{z \mu}$ was found to decrease with an increase in stratification. Whereas there was no appreciable change in $K_{z \sigma}$ and $K_{z \gamma}$.

A significant point to come out of the preceding discussions is that along with the particle settling velocity $(\tilde{V})$, suspended sediment concentration $\left(R i_{\tau}\right)$ is an important parameter that influences the degree of self-stratification in a sediment suspension. Along with different mechanisms that 
Table 5. List of all the experiments of Coleman (1986) used in our study.

\begin{tabular}{cccc}
\hline$R e_{\tau}=3700$ & \multicolumn{3}{c}{$D=0.210 \mathrm{~mm}$} \\
\hline & $R i_{\tau}$ & $\tilde{V}=V / u_{*}$ & $\tilde{V} R i_{\tau}$ \\
\hline 1 & 0.2005 & 0.606 & 0.1215 \\
2 & 0.4583 & 0.606 & 0.2778 \\
3 & 0.6631 & 0.606 & 0.4018 \\
4 & 1.6615 & 0.598 & 0.9936 \\
5 & 2.0140 & 0.605 & 1.2185 \\
\hline
\end{tabular}

Table 6. List of the parameters $K_{z \mu}, K_{z \sigma}$, and $K_{z \gamma}$, calculated from the sediment diffusivity profiles of the cases listed in Table 5.

\begin{tabular}{cccc}
\hline$R e_{\tau}=3700$ & \multicolumn{3}{c}{$D=0.210 \mathrm{~mm}$} \\
\hline & $K_{z \mu}$ & $K_{z \sigma}$ & $K_{z \gamma}$ \\
\hline 1 & 0.2009 & 0.5404 & 1.2555 \\
2 & 0.1862 & 0.5404 & 1.2561 \\
3 & 0.1748 & 0.5435 & 1.2565 \\
4 & 0.1603 & 0.5403 & 1.2569 \\
5 & 0.1560 & 0.5467 & 1.2571 \\
\hline
\end{tabular}

increase sediment diffusivity with the increase in $\tilde{V}$ (van Rijn, 1984; Nielsen and Teakle, 2004) of the suspended sediment, the increase in stratification due to suspended sediment decreases sediment diffusivity. And this effect of selfstratification on sediment diffusivity can explain the anomaly in the expected trend of increasing sediment diffusivity with an increase of $\tilde{V}$ in Fig. 1.

\section{Conclusions}

In the present study sediment concentration profiles from direct numerical simulations of sediment-laden flow through a channel were used, to calculate sediment diffusivity profiles for the channel flow and boundary-layer configuration. The DNS simulations facilitated the evaluation of the effect sediment-induced self-stratification has on sediment diffusivity, without any other competing mechanism playing a role. For the channel flow configuration, an increase in stratification was found to decrease sediment diffusivity in the lower half of the channel and slightly increase sediment diffusivity in the upper half of the channel. For the boundary-layer (open-channel-like) configuration, throughout the channel, sediment diffusivity was found to decrease with the increase in stratification. Though the extent of suppression of sediment diffusivity in the lower half of the boundary layer is appreciably lower than rest of the boundary layer; along the expected lines, the sediment diffusivity profiles reflected the computed turbulence intensity profiles. Observations from the DNS results were vetted against experimental results from Ismail (1952) and Coleman (1986). Sed- iment diffusivity profiles calculated using concentration profiles from Ismail's closed-channel experiments were found to be consistent with the DNS results. Sediment diffusivity profiles calculated using sediment concentration data from Coleman's (1986) experiments were more or less consistent with the DNS results, but the trend of decrease of sediment diffusivity with increase in stratification was erratic. For the present study only one set of Coleman's (1986) experiments was used; it would be interesting to repeat the calculations using data from the rest of Coleman's (1986) experiments and other similar experiments (Cellino, 1998). Through the current study, sediment-induced stratification has been put forth as a plausible explanation for the inconsistencies seen in the expected trends of sediment diffusivity profiles in Fig. 1. There are still other mechanisms (Niño and Garcia, 1998) whose effects on sediment diffusivity have to be eventually evaluated, because a better understanding of sediment diffusivity and the various factors it depends on will eventually help us to ascertain the suspended load in rivers/streams more accurately. At the end of the day, the interaction between suspended sediment and the ambient fluid is highly non-linear and will require further exploration to reveal more of its secrets.

Acknowledgements. We would like to thank Gary Parker, Carlos Pantano and Tzu-Hao Yeh for all the thought provoking and helpful discussions on the topic. This research was possible thanks to the Chester and Helen Siess Professorship in Civil Engineering at the University of Illinois. The support received by the junior author from a CSE Graduate Student Fellowship and the Ravindar K. and Kavita Kinra Fellowship in Civil and Environmental Engineering is gratefully acknowledged. We would also like to thank the two anonymous reviewers for their cogent and insightful remarks, which have contributed towards improving this work. Finally, we would like to thank handling associate editor Francois Metivier for his help during the whole process.

Edited by: F. Metivier

\section{References}

Anderson, A. G.: Distribution of suspended sediment in a natural stream, Trans. Amer. Geophys. Union, 23, 678-683, 1942.

Barenblatt, G. I. and Golitsyn, G. S.: Local structure of mature dust storms, J. Atmos. Sci., 31, 1917-1933, 1974.

Bennett, S. J., Bridge, J. S., and Best, J. L.: The fluid and sediment dynamics of upper-stage plane beds, J. Geophys. Res.-Oceans, 103, 1239-1274, 1998.

Cantero, M. I., Balachandar, S., and Garcia, M. H.: An EulerianEulerian model for gravity currents driven by intertial paritcles, Int. J. Multiphas. Flow, 34, 484-501, 2008.

Cantero, M. I., Balachandar, S., Cantelli, A., Pirmez, C., and Parker, G.: Turbidity current with a roof: Direct numerical simulation of self-stratified turbulent channel flow driven by suspended sediment, J. Geophys. Res., 114, C03008, doi:10.1029/2008JC004978, 2009a. 
Cantero, M. I., Balachandar, S., and Parker, G.: Direct Numerical Simulation of stratification effects in a sediment-laden turbulent channel flow, J. Turbul., 10, p. N27, 1-28, 2009 b.

Cantero, M. I., Shringarpure, M., and Balachandar, S.: Towards a universal criteria for turbulence suppression in dilute turbidity currents with non-cohesive sediments, Geophys. Res. Lett., 39, L14603, doi:10.1029/2012GL052514, 2012.

Cellino, M.: Experimental study of suspension flow in open channels, Doctoral dissertation 1824, Ecole Polytechnique federale de Lausanne, Switzerland, 1998.

Cellino, M. and Graf, W. H.: Sediment-laden flow in open-channels under non-capacity and capacity conditions, J. Hydraul. Eng., 125, 455-462, 1999.

Cellino, M. and Graf, W. H.: Experiments on suspension flow in open channels with bed forms, J. Hydraul. Res., 38, 289-298, 2000.

Coleman, N. L.: Flume studies of the sediment transfer coefficient, Water Resour. Res., 6, 801-809, 1970.

Coleman, N. L.: Effects of suspended sediment on the open-channel velocity distribution, Water Resour. Res., 22, 1377-1384, 1986.

Dutta, S.: Effect of self-stratification on channel flows and boundary layers: a study using direct numerical simulations, MS thesis, University of Illinois at Urbana-Champaign, USA, 2012.

Ferry, J. and Balachandar, S.: A fast Eulerian method for disperse two-phase flow, Int. J. Multiphas. Flow, 27, 1199-1226, 2001.

Garcia, M. H. (Ed.): Manual 110. Sedimentation Engineering: processes, measurements, modelling and practice, ASCE, 46, Reston VA, USA, 2008.

Garcia, M. H. and Parker, G: Experiments on the entrainment of sediment into suspension by a dense bottom current, J. Geophys. Res., 98, 4793-4807, 1993.

Graf, W. H. and Cellino, M: Suspension flows in open channels; experimental study, J. Hydraul. Res., 40, 435-447, 2002.

Ismail, H. M.: Turbulent transfer mechanisms and suspended sediment in closed channels, T. Am. Soc. Civ. Eng., 117, 409-434, 1952.
McLean, S. R.: On the calculation of suspended load for noncohesive sediments, J. Geophys. Res., 99, 5759-5770, 1992.

Mucha, P. J. and Brenner, M. P.: Diffusivities and front propagation in sedimentation, Phys. Fluids, 15, 1305-1313, 2003.

Nielsen, P.: Coastal bottom boundary layer and sediment transport, World Scientific, River Edge NJ, USA, 1992.

Nielsen, P. and Teakle, I. A. L.: Turbulent diffusion of momentum and suspended particles: A finite-mixing-length theory, Phys. Fluids, 16, 2342-2348, 2004.

Niño, Y. and Garcia, M. H.: On Engelund's analysis of turbulent energy and suspended load, J. Eng. Mech-ASCE, 124, 480-483, 1998.

Rouse, H.: Modern conceptions of the mechanics turbulence, T. Am. Soc. Civ. Eng., 102, 463-543, 1937.

Rouse, H.: Experiments on the mechanics of sediment suspension. Proceedings, 5th International Congress for Applied Mechanics, Vol. 55, 550-554, John Wiley \& Sons, New York, 1938.

Schlichting, H. and Gersten, K.: Boundary Layer Theory, SpringerVerlag, 8th Edn., 2000.

Segre, P. N., Liu, F., Umbanhowar, P., and Weltz, D. A.: An effective gravitational temperature for sedimentation, Nature, 409, 594597, 2001.

Shringarpure, M., Cantero, M. I., and Balachandar, S.: Dynamics of complete turbulence suppression in turbidity currents driven by monodisperse suspensions of sediment, J. Fluid Mech., 712, 384-417, 2012.

Smith, J. D. and McLean, S. R.: Spatially averaged flow over a wavy surface, J. Geophys. Res., 82, 1735-1746, 1977.

Vanoni, V. A.: Transportation of sediment by water, Trans. AGU, 3, 67-133, 1946.

Van Rijn, L. C.: Sediment Transport, part II: suspended load transport, J. Hydraul. Eng.-ASCE, 110, 1613-1641, 1984.

Wright, S. and Parker, G.: Density stratification effects in sand-bed river, J. Hydraul. Eng.-ASCE, 130, 783-795, 2004. 